ISSN: 0213-3563

http://dx.doi.org/10.14201/azafea201921181206

\title{
LA EDUCACIÓN MORAL ANTE EL RETO DE LA SOSTENIBILIDAD
}

\author{
Moral education for the challenge of sustainability
}

Jordi Puig Baguer, Ana Villarroya Ballarín y María Casas Jericó

Universidad de Navarra

Recibido: 31 de mayo de 2019

Aceptado: 27 de junio de 2019

\section{RESUMEN}

Ante los indicadores de insostenibilidad ambiental y social, se busca un enfoque educativo eficaz que contribuya a revertirla. A tal efecto, se propone una educación moral, que no se centre tanto en contenidos como en facilitar que cada estudiante aprenda a buscarlos, hacerlos propios y los traduzca en compromisos de conducta personal. La propuesta educativa se presenta ejemplificada en una asignatura concreta, que sirve de hilo expositivo y ejemplo de aplicación. Tras una breve introducción sobre los indicadores de insostenibilidad, se presenta la planificación, metodología y objetivo de la asignatura. A continuación, se reflexiona sobre dos de los obstáculos a los que se enfrenta la educación moral en la actualidad: la fragmentación del saber y la dificultad para que los aprendizajes teóricos se traduzcan en conductas. Por último, se exploran dos oportunidades para la educación moral que pueden facilitar ese vínculo: la búsqueda de las causas profundas de problemas ambientales y sociales actuales, y el empleo de la belleza.

Palabras clave: educación moral; sostenibilidad; justicia social; justicia ambiental; aprendizaje integrado; naturaleza; belleza.

\section{ABSTRACT}

Current indicators of environmental and social unsustainability point out the need of effective educational approaches that revert those trends. From this viewpoint, we propose a focus on moral education, which does not just 
aim to transmitting academic contents but to helping each student individually explore and acquire them, to originate personal commitments that concern personal behavior. To explain and illustrate this, we describe the example of a specific course. After introducing some sustainability indicators, we describe the planning, methodology and objective of the course. Afterwards, we analyze two obstacles for moral education: fragmented knowledge of the world, and the difficulty of helping the students link knowledge and personal, moral behavior. Finally, we explore two opportunities for helping students build such connection: using sustainability indicators to look for deep causes of current unsustainable behaviors, and using beauty to look for personal answers to such problems.

Key words: moral education; sustainability; social justice; environmental justice; integrative learning; nature; beauty.

\section{INTRODUCCIÓN}

La pérdida de biodiversidad, la explosión demográfica -unida al aumento de consumo per cápita-, la deforestación, la pérdida de suelo cultivable, el crecimiento descontrolado de las ciudades de los países en desarrollo, o el cambio climático son algunos ejemplos de los graves retos y problemas a los que la humanidad se enfrenta en la actualidad (PNUMA, 2016; PNUD, 2018). Y es que, en las últimas décadas los retos y problemas ambientales y sociales no solo han ido en aumento, sino que se han agudizado y acarrean ya consecuencias de dimensión planetaria.

La preocupación por la problemática ambiental comienza a hacerse patente a nivel global a finales de los años sesenta del pasado siglo y pronto da lugar a una serie de cumbres internacionales que llegan hasta nuestros (sirvan como ejemplo la Conferencia de las Naciones Unidas sobre el Medio Humano de Estocolmo en 1972, o la Cumbre para la Tierra de Río de Janeiro en 1992) ${ }^{1}$. En todas estas convenciones se ha venido reiterando la importancia de la educación como herramienta para poner freno al daño ambiental y adoptar un modelo de desarrollo sostenible justo a nivel social y ambiental. A tal efecto, la Educación Ambiental y, más recientemente, la Educación para el Desarrollo Sostenible trabajan con ahínco desde hace décadas para

1. En Puig y Casas (2017, pp. 112-117) puede consultarse una síntesis de las principales propuestas educativas para la adopción de una ética de la responsabilidad recogidas en las convenciones celebradas desde los años 70 hasta la actualidad. 
poner freno a la insostenibilidad. Sin embargo, los logros obtenidos, aunque notables, no siempre han sido lo inmediatos o efectivos que a priori se esperaba (Calvo y Gutiérrez, 2007; Saylan y Blumstein, 2011). Por ello, se estima necesario la búsqueda y el desarrollo de planteamientos educativos que complementen a los ya existentes, contribuyendo así al cambio de valores y actitudes que demanda la crisis actual.

En este contexto de necesario cambio cultural, el presente trabajo recoge una propuesta educativa que tiene como objetivo promover la sostenibilidad a través de la educación moral. Dicha propuesta se explica y ejemplifica a continuación a través de una asignatura universitaria. Partiendo de reconocer la insostenibilidad global social y ambiental del mundo contemporáneo, se subraya la necesidad de una educación moral como respuesta, cuyo planteamiento pueda enlazar con otros temas de importancia educativa propios de cada contexto específico, más allá del estrictamente ambiental. Este enfoque permite salir al encuentro de los estudiantes y de otros docentes para adaptar esta propuesta a cada situación académica particular.

En los apartados que siguen, se presentan la organización, metodología y objetivo de la asignatura (apartado 2). A continuación, se muestran dos obstáculos para la educación moral, que tratan de solventarse en la asignatura (apartado 3). Por último, se exploran dos oportunidades para la educación moral (apartado 4): el buscar las causas profundas de lo que sucede a partir de las consecuencias más visibles o inmediatas, y el empleo de la belleza.

\section{Presentación de la asignatura}

La materia "Naturaleza, Tecnología y Sociedad" ofrece a los estudiantes una vía para buscar y encontrar personalmente, desde cada propia situación académica y profesional, respuestas personales al encuentro -conflictivo o no- de las tres realidades que integran el título de la asignatura, y que están tan característicamente entrelazadas en la configuración y sensibilidad cultural del mundo contemporáneo: la naturaleza recibida, los avances de la ciencia y de la tecnología, y la vida humana en sociedad.

La asignatura está diseñada para que pueda ser ofertada a alumnos de cualquier carrera y curso. En la actualidad se imparte en primer curso de diversos grados de Ingeniería, en sesiones compartidas (I. en Tecnologías industriales, I. Mecánica, I. Eléctrica, I. en Electrónica industrial, I. en Sistemas de telecomunicación, I. en Electrónica de comunicaciones, I. en Organización 
industrial, I. en Diseño industrial y desarrollo de productos, I. Biomédica), así como en cursos superiores, también en aula y sesiones compartidas, de Económicas, ADE, Derecho, Psicología y Pedagogía. No obstante, está previsto que se ofrezca próximamente a estudiantes de otros grados. Los profesores que imparten esta asignatura proceden del ámbito de la Biología y las Ciencias Ambientales y cuentan con notable experiencia en educación y en enfoques interdisciplinares, prácticos y experienciales.

"Naturaleza, Tecnología y Sociedad" forma parte del "Core Curriculum” de la universidad en la que se imparte, que reúne a todas las asignaturas transversales que se imparten en la misma. En consonancia con la propuesta de la "Association for Core Texts and Courses", con estas asignaturas se pretende complementar la formación específica de cada disciplina con una educación humanista y en valores, que ayude a desarrollar el espíritu crítico y solidario de cada persona, y promueva la búsqueda libre de las propias convicciones.

En la asignatura se estudian el lugar y el papel de la ciencia y de la tecnología en la actualidad, las cuales gozan de una presencia social y cultural muy significativa, también en las vidas de los estudiantes. Se aborda el reto de pensar y valorar esta presencia, con el fin de saber participar críticamente en su dirección social y en las elecciones personales que la alimentan. Para lograrlo, se contrastan una selección de desarrollos científicos y tecnológicos significativos de hoy con los valores de la naturaleza y de la vida en sociedad humana, realidades a las que, indudablemente, pertenecemos.

Los valores de naturaleza y sociedad son antropológicamente anteriores a los desarrollos concretos de la ciencia y la tecnología, al ser históricamente preexistentes, y materialmente posibilitadores de toda construcción cultural. Por todo ello, esos valores son a la vez radicalmente fundantes de cómo son los seres naturales y humanos, así como también del deber ser dejado a la libertad de los últimos. Frente a esta, los valores se le ofrecen como guía de conducta no impuesta, pidiendo un respeto que alcanza, por ejemplo, a las múltiples posibilidades de la actividad científica y tecnológica. En este sentido, la actual crisis ambiental parece inseparable de la dirección global concreta que se le ha conferido a los desarrollos científicos y tecnológicos (UNESCO, 2015).

\subsection{Planificación, contenidos y metodología}

La asignatura prevé catorce sesiones presenciales de dos horas cada una. Las seis primeras y la última están dirigidas por los profesores. Las siete 
sesiones restantes son dirigidas por los alumnos, que presentan un trabajo que se les ha encargado.

La asignatura comienza reflexionando sobre la naturaleza (sesiones 1 y 2 ) a la que se suma posteriormente -y de forma entrelazada con esta-, la sociedad (sesiones 3 y 4), precisamente por su condición de valores fundantes. A esto sigue la reflexión sobre ciencia y tecnología (sesiones 5 y 6), que cierra la primera parte de las clases presenciales. Estos temas son abordados siempre en forma dialogada, tomando ocasión de aspectos como los que se recogen en la Tabla 1. En relación con los contenidos que se presentan en dicha tabla es preciso realizar una precisión: no se ha tratado tanto de fijar cuáles puedan ser los contenidos de la educación moral con la que se propone salir al paso de las crisis de sostenibilidad, como de sugerir una vía adecuada, y ya en cierta medida contrastada por la experiencia, de promover esa educación en otro sentido que el de fijarse primariamente en sus contenidos en lugar de en la libertad y el compromiso del estudiante.

Como se ha adelantado, los valores de naturaleza y sociedad señalan de algún modo, en ocasiones difícil de discernir, el deber ser deseable o exigible a los avances científicos y a los desarrollos tecnológicos, los cuales, por otra parte, se distribuyen muy desigualmente en el mundo (UNESCO, 2015). Estos valores, por más que pueda ser veladamente, alcanzan también el deber ser de la propia conducta respecto a ellos, especialmente en una sociedad consumista, donde el consumo personal incide en ellos. Se busca así que el estudiante ponga en relación lo que sucede al mundo desde una perspectiva global con la propia conducta, y evitar un anonimato irresponsable o una disolución de la parte personal de culpa o deber en el todo social. Además, el deber ser que solicitan esos valores fundantes, la naturaleza y la sociedad, se refiere a ámbitos de la antropología que van más allá de las meras relaciones entre ciencia, tecnología y ser humano, al interpelar a la conducta moral en su raíz (Puig y Casas, 2017).

El diseño de la primera parte de la asignatura propone, por tanto, que usar como referencia los principales valores naturales y sociales ofrece un buen punto de apoyo y arranque para pensar y valorar la ciencia y la tecnología adecuadamente, incluyendo el acceso a ellas y la conducta moral que reclaman a cada uno de sus beneficiarios. Busca desarrollar el hábito de ir y volver a los fundamentos naturales y sociales de la existencia del mundo y del ser humano, para repensar críticamente desde ellos las manifestaciones del encuentro entre ambas realidades, en armonía o conflicto, que requieren un nuevo examen a la vista de la insostenibilidad. 
El diseño de la asignatura permite también, y siguiendo su propia óptica, abordar supuestos clave de la antropología que pueden estar en exceso olvidados en la práctica. En especial los de carácter natural y de justicia social, que parecen estar desplazados por un exceso de atención a lo artificial, o al bienestar de unos pocos, que son los que más deciden cómo funciona el mundo (Escámez, García y Pérez, 2003). En este sentido, se diría que la crisis ambiental y la injusta desigualdad en el acceso a los bienes de la tierra testifican ese olvido práctico, que reclama promover un nuevo compromiso cultural y personal con lo natural y el interés común, al que se invita desde la asignatura a los alumnos.

Volviendo a la planificación de la asignatura, tras las sesiones presenciales se llevan a cabo las exposiciones de los trabajos realizados en equipo por los alumnos (sesiones 7 a 13). Estas sesiones se dedican al estudio y reflexión sobre los Objetivos de Desarrollo Sostenible de Naciones Unidas (ONU, 2017), que reúnen de manera muy integrada cuestiones de naturaleza, tecnología y sociedad en su impulso por promover un mundo más saludable, justo y equitativo. Finalmente, en la última sesión (número 14), los profesores realizan una recapitulación de todo lo precedente, que se articula en torno a la experiencia de la belleza.

Además de las sesiones presenciales y el trabajo en grupo, la asignatura prevé otras herramientas educativas. De una parte, la entrega individual de ensayos sobre el desarrollo efectivo de cada una de las sesiones presenciales (1 a 6), sintetizando el contenido de la clase y añadiendo una reflexión personal sobre las cuestiones que surgieron. Se estima que este modo de proceder permite fijar mucho más un aprendizaje -que además es y se hace propioque la realización de un examen de contenidos, precisamente al poner en relación los temas tratados con el propio modo de entenderlos y valorarlos (Espinosa, 2016). El mismo ejercicio, pero acerca de lecturas o documentales relacionados con los temas tratados en clase, se propone de forma adicional a aquellos alumnos que quieran mejorar sus calificaciones.

La capacidad educativa de los ensayos se ve alimentada por la revisión y anotación personal de los mismos por parte de los profesores, lo que a su vez es una forma de evaluación continua. El ejercicio de redactar comentarios personalizados a cada alumno sobre sus propias reflexiones ayuda al profesor a detectar cómo han interiorizado los estudiantes los temas tratados en cada sesión y a apoyarles en ese trabajo, a través de un diálogo académico personal efectivo. Por último, se ofrece también a los alumnos que quieran optar a las mejores calificaciones, la posibilidad de escoger y certificar la realización de una labor de voluntariado que esté en relación con los temas académicos que se desarrollan a lo largo de la asignatura. 


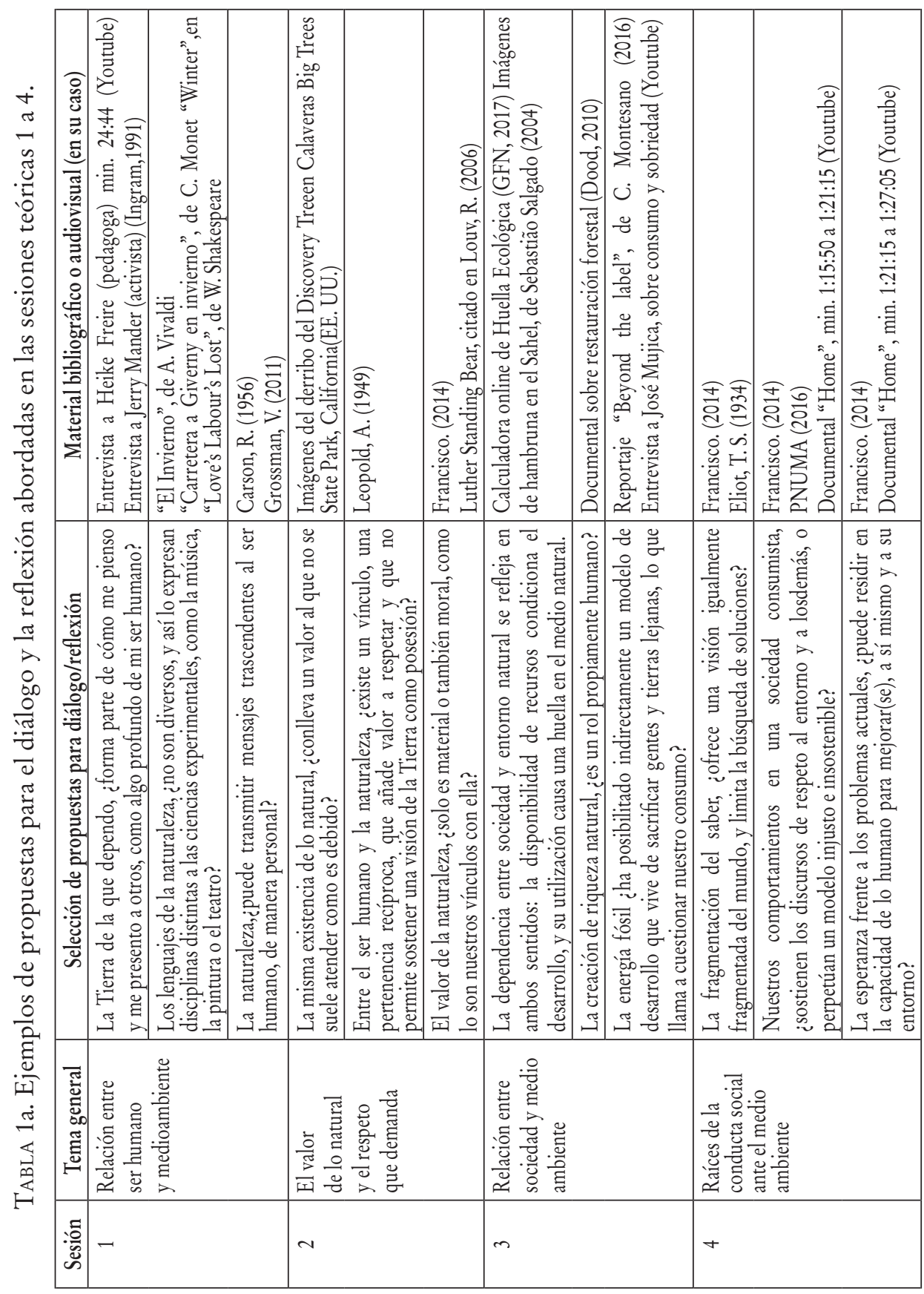




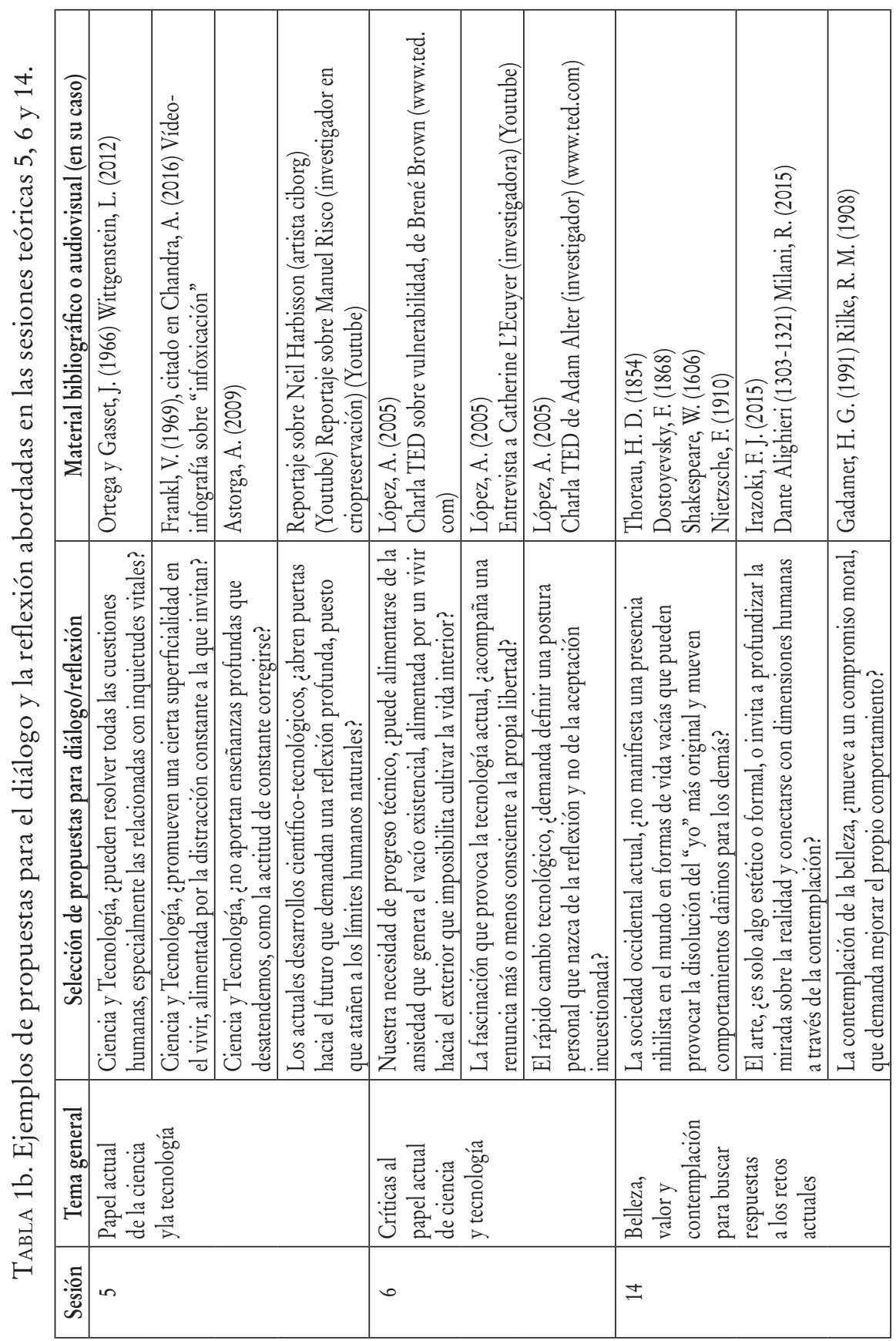




\subsection{Objetivo: una educación moral}

El orden de temas expresado en la primera parte de la asignatura (Tabla 1, apartado 2.1.) no debe hacer pensar, como ya se ha adelantado, que la reflexión sobre ciencia y tecnología sea el término final al que se desea llegar impartiéndola. Más bien, se emplea la visibilidad e integración en el mundo de hoy de la ciencia y la tecnología -y en la experiencia de los estudiantespara promover un marco de aprendizaje antropológico y moral desde ellas -y desde esa experiencia personal del alumnado. Se busca, con el enfoque que se da a la asignatura, desarrollar el hábito de pensar y valorar moralmente, partiendo de bases antropológicas lo más firmes y universalmente aceptadas por los alumnos posible, las relaciones con la naturaleza y entre los seres, así como las consecuencias que las propias decisiones libres personales tienen sobre los valores naturales y sociales.

Si se apunta a posibilitar esta educación moral, como objetivo, es porque se considera especialmente eficaz - por ir directamente a la raíz del problemay necesario incidir en ella para hacer frente a los retos del mundo contemporáneo. Sin embargo, no es objeto de la asignatura proponer unos supuestos contenidos concretos de la moralidad, un detallado cómo hay que conducirse. Esta tarea, en la que tiende a incidir mucho la educación ambiental (Álvarez y Vega, 2009), se deja para los alumnos. Se estima que este modo de enfocar las cosas - perfectamente válido y legítimo en muchos contextos educativossería precisamente contraindicado para una correcta educación moral, que es la que se persigue promover, y que debe estar más arraigada en el corazón que en una mera voluntad educada, si puede adjetivarse así. Se entiende que toda educación moral debe insistirse (a sí misma) en presuponer, respetar y alimentar la libertad personal. Por tanto, es más coherente promover una educación moral que limite los contenidos específicos a los mínimos indispensables desde los que debe proponerse su progreso, y que cada educador juzgue cuáles son los apropiados para su ámbito.

Como ejemplo de los contenidos mínimos de los que se puede partir, se recurre con insistencia en la asignatura a diversas intuiciones e injusticias ambientales y sociales detectadas como tales por la sensibilidad contemporánea (Tabla 1). Se estima que lo fundamental para el aprendizaje que se propone la asignatura es que, a partir de esos contenidos (pueden ser otros...) se fomente que los alumnos discutan y descubran por sí mismos sus deberes, y adopten así sus compromisos. Se busca evitar en lo posible que los estudiantes elijan conductas pre-escogidas para ellos. La asignatura misma es optativa, ya de 
entrada. Una vez matriculados, les obliga a trabajar, pero de modo que el trabajo sea en gran medida voluntariamente escogido y dirigido (ver apartado 2.1).

Se pretende, en definitiva, ofrecer herramientas para que cada estudiante vaya concluyendo los contenidos de la moralidad para su conducta, cuando entra en contacto con la realidad que se le presenta, pensada y reflexionada académica e individualmente. Se promueve que el estudiante busque esos contenidos en contacto con el ser y valor de cada realidad natural, humana y social, que exigen respeto de por sí. Por otra parte, se le anima a descubrir que ese respeto no es una llamada solamente de carácter genérico, la que vincula a todos por igual. Tiene también una dimensión personal en exceso olvidada por la educación que, sin anular la anterior, es específica de cada persona y solamente ella puede descifrar en el silencio de su corazón (Puig y Echarri, 2018). De ahí que, por ejemplo, ante la experiencia de la pobreza distintas personas puedan responder de modos tan variados como legítimos y libres: recortando el propio consumo para ayudar más, colaborando con una ONG, asistiendo a personas necesitadas en un comedor social, viajando a campos de refugiados, etc.

La profundización académica del contacto con la realidad, a veces oculta en sus dimensiones más valiosas, se fomenta con el voluntariado (Rincón, 2010) y a través del diálogo en las clases y la reflexión en los ensayos que se encargan semanalmente a los estudiantes, sobre los temas de interés que se presentan a su consideración. Los indicadores ambientales y sociales que se traen al aula muestran que algo serio en nuestras sociedades no anda bien. Se busca de esta manera que surja el aprendizaje y el compromiso que estas realidades requieren como respuesta.

El estudio de las relaciones con la naturaleza, y entre los seres humanos en su seno, así como de las consecuencias que las propias decisiones libres tienen sobre los valores naturales y sociales, se estiman especialmente de interés como punto de reflexión. El modo de vida actual tiende a ocultar de la experiencia cotidiana muchas de estas relaciones -que pueden darse por supuesto, considerándolas válidas acríticamente- y de las consecuencias ocultas a la percepción que acarrean. Ambas son las que se proponen como puntos de reflexión en clase. Por tanto, se plantea que, ya sea por inconsciencia o por falta de concienciación, se pueden estar omitiendo responsabilidades, alimentando así una triple insostenibilidad en la sociedad contemporánea: ambiental, social y moral. De estas tres, se considera que la insostenibilidad moral es la más oculta y a la vez la que más va a la raíz del problema, como ya se ha sugerido más arriba. De ahí que, buscando la mayor eficacia educativa posible, la asignatura se presente, en su raíz y fondo, como una oportunidad de aprendizaje moral. 
El modo de vida contemporáneo, a la vez que oculta la insostenibilidad en sus causas vinculadas a las conductas cotidianas colectivas y en sus efectos externalizados, está desarrollando, sin embargo -como se ha adelantado al principio de este apartado- una elevada capacidad de captar ciertas consecuencias, impactos negativos o expresiones concretas de insostenibilidad ambiental y de la injusta desigualdad social. Partiendo de la sensibilidad de los estudiantes ante esas consecuencias, y yendo hacia atrás en la cadena de la causalidad, se pueden hacer menos invisibles las causas más fundamentales en las que arraiga la injustica ambiental y social. En último término, siguiendo esa cadena de causalidad, se logra hacer más visible la insostenibilidad moral antropológica en la raíz de esas conductas -acaso personales- que, de un modo previo a la reflexión, no se percibían conectadas con la injusticia que se deplora. Se espera que esa evidencia sea una eficaz invitación personal a cambiar por propia convicción la conducta, que sería el mejor logro educativo de la asignatura. No obstante, ese logro se deja en manos de cada estudiante, tras asegurar el mínimo exigible de su trabajo personal y en grupo, y el acercamiento académico a la necesidad de compromiso. La educación moral, por su propia naturaleza, no puede medir académicamente gran parte de los fines que desea que los estudiantes alcancen: es una educación, en gran medida, para la vida que vendrá.

\section{OBSTÁCULOS PARA UNA EDUCACIÓN MORAL EFICAZ}

A continuación, se presentan dos aspectos que, a juicio de los autores, dificultan el desarrollo de una educación moral efectiva: el conocimiento fragmentado de la realidad, y la necesidad de vincular -en el contexto del aprendizaje contemporáneo-, la teoría y el desarrollo o cambio de comportamientos personales. La asignatura trata de suplir estos obstáculos incluyendo aportes de diversas disciplinas (por ejemplo, ciencias experimentales, literatura, o filosofía), y fomentando la reflexión personal sobre la propia conducta.

\subsection{El conocimiento fragmentado del mundo}

Las diversas realidades del mundo (material natural o artificial, intangible o cultural...), acaso distinguibles teóricamente y en origen, se hallan sin 
embargo integradas de un modo indisociable en esa misma realidad bajo estudio, de enorme complejidad. Lo material, moral, tangible e intangible está íntimamente conectado: tal es el carácter de la realidad. El estudio de la interrelación entre naturaleza, sociedad y tecnología así lo permite mostrar y recordar de un modo particularmente cercano a la experiencia de los alumnos, paso a paso, y a partir de la creciente sensibilidad moral ambiental y social del tiempo presente.

Dada esa complejidad del mundo, un acercamiento exclusivamente experimental a la realidad (no se hace referencia ahora a la experiencia subjetiva de los alumnos, sino a la experimentación propia del método científico) es enriquecedor, pero no permite alcanzar la plenitud de valor que esa realidad atesora, ni movilizar las potencias del corazón que la ciencia por sí sola parece no poner en marcha. Es posible que la fama de practicidad en la enseñanza y el aprendizaje de las llamadas ciencias experimentales sea menos deseable que el entusiasmo que sus descubrimientos deberían promover en el corazón del estudiante. De lo contrario, la educación científica puede pavimentar un camino hacia la instrumentalización de la realidad natural (Macfarlane, 2016), que acabe impregnando también el desarrollo de las aplicaciones técnicas, su uso y las mismas relaciones sociales.

De no primar el entusiasmo por el descubrimiento, en la educación de las ciencias experimentales, tal vez estemos equivocando el modo en que las enseñamos. Estas ciencias no son experimentales para expulsar al corazón personal de aquellos que profundizan en ellas. Lo son para asegurar la capacidad de réplica -de falsación, diría Karl Popper (Artigas, 1979), uno de los más destacados filósofos de la ciencia del siglo XX-de los experimentos, en una búsqueda sin término de la verdad. Esa experimentación, si no se la mutila y desconecta, desvela -integrada en la realidad- la belleza también científica al corazón atento a intereses que vayan más allá de una mera instrumentalización de lo investigado, y promuevan un asombro admirativo y respetuoso (Carson, 1956).

Por esta razón, la vía científico-experimental por sí sola, indebidamente aislada, desconectada del resto de lo humano, no permite reconocer ni enfrentar toda la gravedad de los conflictos que puede causar la conducta humana, personal y colectiva. Tampoco moviliza todas las fuerzas para contrarrestarla, que no se limitan a las de la inteligencia y la voluntad, sino que demanda un involucrarse del corazón que trascienda "intelectualismos descomprometidos o voluntarismos forzados que expresen la mutilación del corazón en la educación” (Puig y Casas, 2017, p. 123). Y esto se estima de destacada importancia para la educación moral. 
La conducta arranca, un tanto paradójicamente, de decisiones intangibles en su origen y ya -incluso sin que se manifiesten materialmente- de carácter moral. Solamente tras la decisión del corazón, al actuar, puede que se exprese la dimensión tangible de la conducta. Pero el carácter moral no se limita a lo tangible que se causa en la dimensión material, porque arraiga de origen en el intangible de la libertad del corazón humano que decide la conducta. Siendo valiosa científica y moralmente, la ciencia experimental puede malentenderse hacia considerar desproporcionadamente, en exceso o exclusivamente los valores tangibles, en detrimento de los intangibles. De forma análoga, un exceso de concentración en lo tangible puede hacer olvidar intangibles previos sobre los que sería más decisivo (y difícil) actuar.

No se pretende con la afirmación anterior inducir al error de minusvalorar el papel moral que aportan per se las ciencias experimentales, y que ellas mismas en primer lugar deben abrazar. El auge del compromiso ambiental promovido con el avance de la disciplina de la Ecología atestigua ese papel moral en las últimas décadas, por ejemplo. En cuanto que descubren -por vía experimental replicable- valores reales, como por ejemplo las condiciones de salubridad de las aguas de un río, las ciencias experimentales señalan el camino debido de compromiso moral: no nos está permitido moralmente -aunque sí de hecho, a la vista está- usarlas de un modo que sabemos que contamina y reduce su calidad, y que se puede evitar. Desatender los valores descubiertos experimentalmente, así como los compromisos morales que solicitan, es lo que está llevando a la insostenibilidad ambiental.

La minusvaloración del carácter moral de la ciencia, por desgracia, se ve alimentada también con frecuencia desde una mal entendida o mal ejercida educación humanística (Puig, Echarri y Casas, 2014), cuando no se presta atención a los valores que las ciencias del medio ambiente van descubriendo, con métodos tanto experimentales como más propios de las ciencias sociales. Por desgracia para la educación moral, se ha podido afirmar lo siguiente:

Las ciencias experimentales y técnicas no abren suficientemente todavía, con naturalidad y rigor, su mundo empírico al pensamiento, la cultura, el espíritu y las cuestiones universales y permanentes sobre el ser humano, su valor y su sentido. Las artes o las humanidades, por su parte, no se acaban de creer que las ciencias ambientales aporten riquezas humanamente relevantes, ni que el medio ambiente deba ser una cuestión central también para las disciplinas que cultivan el espíritu y la convivencia humana. He aquí la fragmentación del saber académico y de la cultura, ante la cuestión del medio ambiente (Puig y Casas, 2017, p. 108). 
Esta fragmentación de disciplinas, más preocupante cuando separa a ciencias y humanidades en dos grandes polos académicamente distanciados, puede dañar especialmente la posibilidad y consistencia de una educación moral. De una parte, cuando la ciencia y la tecnología, o su disfrute consumista, se desentienden de un autocontrol de carácter ético, exigido por el valor mismo de la realidad a la que afectan en su desarrollo y aplicación, y cuyo valor ayudan a descubrir con su modo de conocimiento. Esta cuestión es abordada en las sesiones 5 y 6 del temario de la asignatura (Tabla 1). De otra, cuando la educación humanística no da suficiente relevancia a los contenidos de la ciencia que, al avanzar, descubre valores naturales y humanos, humanísticos, a respetar. Este descubrimiento de valores es notablemente visible hoy desde la Ecología y las ciencias del medio ambiente. La sensibilidad que ha surgido con su auge sugiere que muy errada está la conducta colectiva hacia el medio ambiente, natural y social, cuando se basa en restar globalmente valor a la riqueza que la hace posible, a la vez que crece la desigualdad entre los seres humanos (Bauman, 2016).

Los avances de las ciencias ambientales -debidamente seleccionados, dimensionados y adaptados- deberían ser incluidos en toda educación que se pretenda realmente humanística, pues desvelan valores morales que fundan la posibilidad y la misma constitución de lo humano. De traerlo a consideración y recordarlo se encargan las sesiones 1 y 2 de la asignatura (Tabla 1). Es también relevante, en este sentido, el olvido de los conocimientos y valores acerca de las condiciones de vida digna y la falta de justicia distributiva en el mundo que señalan las ciencias sociales. De abordarlo se encargan las sesiones 3 y 4 del temario (Tabla 1 ).

\subsection{El modo de aprendizaje contemporáneo}

La fragmentación académica del saber es, como se ha mencionado anteriormente, un obstáculo para una adecuada educación moral; pero no es el único. Se quiere ahora incidir en otro obstáculo al que, desde la asignatura y como refleja su planificación, se le otorga una notable importancia: la necesidad y dificultad de vincular en lo posible, en el modo de aprendizaje contemporáneo (Bauman, 2007), teoría y práctica. Se considera aquí práctica en el sentido de conducta escogida, haciendo referencia al aprendizaje moral que se propone la asignatura. No se refiere, por tanto, a la unión de aprendizaje teórico y capacitación para la acción técnica (un mero hacer prácticas), sino 
a la necesidad de promover que se vincule, por propia decisión, lo conocido con la conducta moral.

Saber es, en gran medida, descubrir dimensiones de la realidad -de sus modos diversos de ser y de relacionarse- que reflejan múltiples valores a respetar con la propia conducta libre, la cual siempre tiene una dimensión moral vinculada a esa libertad. Así, y retomando el hilo ambiental, a medida que la Ecología se ha desarrollado, debería haber ido promoviéndose en la sociedad un paralelo compromiso con el medio ambiente. En buena medida se ha dado, como se ha comentado en la introducción, pero no en todo el grado debido. De ahí la actual y creciente insostenibilidad global.

La deseable conexión entre conocimiento y conducta moral no sólo es propia de los aprendizajes ambientales. A medida que progresa el conocimiento aportado por cualquier disciplina académica, aparecen nuevos valores que pueden reclamar más respeto del que reciben de hecho con las conductas. Por esta razón, los temas de sostenibilidad y justicia ambiental y social desde los que se busca la educación moral en esta asignatura podrían ampliarse, modificarse. Los profesores han escogido partir de aquellos enfoques o temas en los que vienen trabajando desde hace años, para asegurar el adecuado nivel académico por su parte. Pero siempre se han propuesto llegar, desde ellos, a la raíz más profunda y común posible de los retos en torno a los que se reúne al fin el mundo académico en sus propuestas. Y ello es posible gracias al planteamiento del objetivo de la asignatura: la búsqueda de una educación moral que vaya a las raíces más profundas de lo que se debe cambiar.

Por tanto, el enfoque que se presenta pueda servir de inspiración para otras propuestas educativas que, partiendo de realidades alternativas, puedan confluir en la búsqueda de una educación moral. Se podría, por ejemplo, partir de temas como las redes de extorsión y prostitución, la violencia hacia las mujeres, la venta de armamento a países en desarrollo, las trabas del comercio internacional a los países pobres... Son todos ellos temas de enorme importancia y actualidad que, por limitación de tiempo y por no haber sido académicamente investigados por los profesores de una forma más detenida, no se abordan directamente en la asignatura salvo en la medida en que los alumnos los quieran traer a consideración.

Ante estos enormes retos, la educación (en especial la de grado universitario) tiende a asegurar conocimientos y capacitaciones, acaso dejando demasiado de lado la necesidad de orientarlos hacia sus últimas y más relevantes consecuencias o demandas, que están resumidas en el compromiso moral. Es en el corazón de cada estudiante -o profesor-donde se dilucida ese compromiso... 
o su falta. Y si se dejan de lado el corazón y el compromiso, esto sucede, en parte, porque la educación de hoy tiende a favorecer demasiado el aprendizaje de la realidad bajo la óptica dominante del interés, de su función instrumental para el ser humano (Macfarlane, 2016). Se tiende a enseñar a hacer e incluso a ir más allá de todo lo que técnicamente es posible. Y esa actitud configura en notable medida la mentalidad contemporánea, de carácter marcadamente utilitarista en las relaciones que la tejen (Bauman, 2016).

La ausencia o debilidad del vínculo entre conocimiento y moralidad se pone de manifiesto en especial cuando es el propio conocimiento el que instrumentaliza y somete la realidad a la que debería proteger con el respeto. Los desarrollos intelectuales científicos y tecnológicos tienden con frecuencia a desentenderse, por esa vía instrumental, del valor en sí mismo de lo conocido. Se olvida, paralelamente, fomentar el respeto de ese valor. Este fenómeno explica, por ejemplo, que la física nuclear acabe al servicio de la tecnología de guerra potencialmente más devastadora que se ha inventado hasta el momento. Más nos valdría aprender a admirarnos de la belleza de las estrellas, demasiado olvidadas, en las que se forjaron los elementos que hoy, tras millones de años, nos constituyen. Afortunadamente, aplicaciones como la medicina nuclear permiten mantener la esperanza, de signo opuesto, en que se puedan subrayar más otros modos de responder al progreso en el conocimiento.

La asignatura que aquí se presenta aborda esa nueva expresión de fragmentación, la desconexión entre conocimiento y responsabilidad moral, insistiendo en el vínculo entre ambas. Para promover el restablecimiento de esa vinculación, además del enfoque empleado en las sesiones de la primera parte de la asignatura (apartado 2.1.), se exige a los estudiantes preparar en grupo, exponer y debatir en clase, uno de entre los 17 Objetivos de Desarrollo Sostenible de Naciones Unidas (sesiones 7 a 13, Tabla 1, apartado 2.1.). Además, se ofrece a los estudiantes que aspiran a obtener las máximas calificaciones la posibilidad de realizar una labor de voluntariado, que esté en relación con los temas académicos que se desarrollan a lo largo de la asignatura. Estos dos ámbitos de iniciativa y compromiso se suman al carácter debatido de las sesiones teóricas 1 a 6 , que les invitan a intervenir y discutir constantemente con el material que se aporta en ellas, y que además les aportan ideas sobre cómo preparar su presentación.

A todo ello hay que añadir los trabajos semanales sobre el desarrollo efectivo de las clases teóricas (sesiones 1 a 6 ) y sobre las lecturas que se proponen tras las sesiones 1 a 6 . En los primeros, recordemos, se pide a los alumnos que 
resuman y reflexionen sobre el transcurrir de la clase desde su punto de vista; en los segundos, que comenten lecturas escogidas en relación con los temas tratados. Se busca así, de todas estas maneras, promover que los alumnos puedan realizar una aportación creativa y significativa a la asignatura, que favorezca su compromiso desde una reflexión académica dialogada, pensada y propia, con la ayuda posible del profesor. Se busca promover, en suma y como se viene recordando, la educación moral personal.

\section{OpORTUNIDADES PARA LA EDUCACIÓN MORAL}

De modo análogo al apartado anterior, a continuación se abordan dos cuestiones que, a juicio de los autores, favorecen el desarrollo de una educación moral efectiva: el estudio de las relaciones y consecuencias, y el empleo de la belleza.

\subsection{El estudio de relaciones y consecuencias}

La asignatura da por supuesto que, de una forma implícita, cada cultura facilita como por ósmosis la incorporación, en gran parte inconsciente, de no pocas normas valiosas de conducta en las personas que la comparten. Estas normas encierran una importante dimensión y construcción moral. Gracias a ellas, actualmente no tiene que discutirse, por ejemplo, la corrupción moral que encierra la aceptación de la esclavitud.

Para alcanzar una educación moral efectiva, esta ha de complementarse con aquellas exigencias de justicia que la propia cultura puede no estar encarnando. Esto es especialmente visible hoy en la insostenibilidad de no pocas relaciones con el medio ambiente, y en las desigualdades entre diversos sectores sociales y naciones. Estas relaciones insostenibles, injustas, pueden ser tomadas como oportunidades educativas (Puig y Casas, 2017). Así se hace en la asignatura, que aprovecha la sensibilidad contemporánea que encarnan los estudiantes al respecto. Sin embargo, no basta con dar por supuesta esta sensibilidad en materia ambiental y social para promover el progreso moral y el compromiso que requiere; es preciso promover explícitamente la reflexión sobre las relaciones entre seres humanos y entre estos y la naturaleza.

El conocimiento y reflexión sobre lo que está sucediendo en la realidad está en relación directa no solamente con el ejercicio y crecimiento de la 
libertad personal, sino con el progreso hacia unas sociedades y culturas más justas. Se busca, al estudiar esas relaciones, fomentar no sólo el conocimiento de lo que se presenta, sino también el compromiso personal, respetando la libertad y promoviendo la creatividad.

A lo largo de toda la asignatura se reflexiona sobre el encuentro, la intersección o relación, las diferencias y los posibles conflictos y soluciones que se pueden dar entre la realidad dada -es decir, no debida al ser humano y que pertenece al ámbito de lo que llamamos natural-y la realidad sí debida a la presencia y actividad humana. La presencia y la actividad humana abarcan, por ejemplo, las elaboraciones materiales indiscutidamente artificiales, cualquier grado de influencia ejercida sobre la naturaleza material, y también las aportaciones humanas intangibles, como el carácter que le da a un paisaje haber albergado un reconocido hecho histórico o cultural. Todas ellas son examinadas a lo largo de la historia y a través del territorio, en distintas sociedades y culturas humanas a través de casos y temas escogidos que conecten con la sensibilidad moral de los estudiantes.

Al hilo de esos casos y temas, se reflexiona sobre cómo la presencia y la actividad humana se hacen patentes en los paisajes, en mayor o menor grado humanizados. La tierra, a cualquier escala -sea en el interior de un barrio, de una ciudad, región, país o continente- puede expresar un envidiable equilibrio que mantener y del que aprender, o revelar graves desequilibrios y conflictos con los valores naturales o sociales, que se deben tratar de resolver. $\mathrm{Al}$ detectarse aciertos y desajustes en este encuentro (impactos ambientales y sociales percibidos como particularmente graves), se identifican puntos a partir de los que iniciar con los estudiantes la búsqueda retrospectiva y el reconocimiento de valores, naturales y sociales, acaso injustamente desatendidos o incluso afectados.

Se sobreentiende, con este modo de proceder, que el valor -en buena parte inexpresable en términos de mercado, el cual es siempre insuficiente para reconocer y valorar plenamente un valor natural o humano- está fundado de algún modo inalcanzable en una realidad que reclama respeto. Y que ese valor, en parte insondable pero capaz de llamar al corazón, puede estar siendo desatendido, desprotegido o dañado y solicita un compromiso moral -que reúna y enlace sensibilidad, inteligencia y voluntad- para protegerlo en su fragilidad.

Este modo educativo de proceder, que busca reunir tres grandes dimensiones humanas (sensibilidad, inteligencia y voluntad) a través del examen de las relaciones y, particularmente, de la conflictividad reconocida o del valor 
descuidado o dañado, busca promover la educación a través del despertar moral del corazón. Un despertar que se ayuda a interpretar como llamada a la que responder personal y escogidamente, con la misma libertad personal que es interpelada. Se quiere evitar, como se ha adelantado, un aprendizaje moral basado solamente en asimilar, acaso acríticamente, las normas de conducta socialmente establecidas. Se busca, en cambio, que la conducta -en particular en torno a los valores y las injusticias- se asiente en el respeto a valores personalmente reconocidos, en parte examinando cuándo son pisoteados y deben ser rescatados a través de un compromiso personal que cree -desde el corazón- una cultura de respeto. Asimismo, se pretende que el alumno aprenda a buscar y reconocer el valor que toda belleza encierra y señala.

\subsection{El empleo de la belleza}

La última sesión de la asignatura (la decimocuarta) está pensada para recapitular todos los temas que se han abordado, pero desde una nueva perspectiva: la belleza. La motivación para centrar esta sesión final en torno a la belleza reside en que se estima que esta encierra un fuerte potencial educador moral, e incluso una invitación a un estilo educativo de respeto por la libertad personal.

Se ha mencionado que la educación moral por la que se opta, en la que se cree, no es la de proporcionar primariamente unos supuestos contenidos o normas de conducta específicos a los alumnos. Y que se busca, en cambio, dotarles de nuevas herramientas o recursos para que ellos mismos descubran esos contenidos y normas, personalmente y en contacto con el valor de lo real. Unas herramientas que, se desea, perduren más allá de la vida académica de estudiantes. No se actúa así de abiertamente movidos por un enfoque relativista, sino por el deseo de promover una conducta basada más en la búsqueda personal de valor en la realidad misma que en la supuesta autoridad delegada indebidamente en otros, o meramente acordada acríticamente. Y uno de los modos de acceder a esa realidad en sus dimensiones más profundas se estima que puede ser la belleza contenida en lo real. La belleza, no siempre fácil de ver, sería una movilizadora del corazón potencialmente inmejorable.

Desde el punto de vista de la educación moral, delegar la decisión de la propia conducta no tiene sentido ninguno. A no ser bajo circunstancias muy excepcionales que no constituyen el núcleo de la educación moral que se busca promover. Por ejemplo, la decisión de intervenir o no a un ser querido, cuando alguien no experto se apoya en la opinión del médico ante un 
caso difícil. Se estima, fuera de casos análogos a este que se menciona, que la educación moral ha podido caer con demasiada frecuencia en el error de ser delegada en exceso en una autoridad externa, cuando no hacía ninguna falta ni era tan siquiera recomendable. Esta educación, basada en una autoridad delegada, no habría pasado en la medida debida por el corazón de cada persona a la que se le ofrecía. Precisamente ese error habría fomentado, con el tiempo y "a posteriori", no pocos rechazos a autoridades que se aceptaban en el pasado de una forma demasiado acrítica. Esa repulsa también hoy puede que se esté expresando en el rechazo sordo y contenido, como subterráneo, a ciertos elementos de lo que se percibe como políticamente correcto, secretamente censurados desde muchos corazones por más que sean considerados correctos y dominen el discurso público. Se logra así que se viva de un modo opuesto al que se manifiesta. Una actitud que, por desgracia, parece muy presente en el contraste actual que existe entre sensibilidad ambiental manifestada y conducta real de consumo (Chuvieco et al., 2017).

La crisis de autoridades morales, en parte causada por las que pudieron ahogar en el pasado la libertad, ofrece, en gran medida, una oportunidad liberadora. Hace falta proponer modos de encontrar personalmente las respuestas a los interrogantes sobre el "deber ser" de la propia conducta, que salven el riesgo de caer en el relativismo descomprometido o la incomunicación. Se propone que la belleza ofrece la manera acaso más profunda y estimulante de obtener esas respuestas, que siempre serán personales, abiertas a la compañía y buscadas en la realidad, previniendo así en lo posible enfoques tanto relativistas descomprometedores como autoritarios.

La belleza ofrece un lenguaje en cierto modo universal. Al ser captada como tal por esa dimensión personal misteriosa a la que llamamos corazón, hace que quienes la ven se sientan y reconozcan libres. Además, cada uno que la percibe descubre que otros ojos la pueden ver también como los propios. Al reconocerla por igual, esas miradas se reconocen semejantes, hermanas, sin perder su experiencia de libertad moral. Se tejen así consensos de respeto y de encuentro entre diversos. Se propone que la belleza no solo revela en su lenguaje propio lo más profundo de los valores que anidan en la realidad, sino que también, debido a la universalidad de su lenguaje, promueve la fraternidad de quienes, distintos, la buscan y se encuentran en ella.

La belleza solicita, para ser percibida en todo su alcance moral, aprender a mirar de otro modo al habitual. Pide un acercamiento contemplativo, detenido, no meramente instrumental o aplicado, en el que el propio interés pase a segundo plano, y se disponga al cambio de conducta, al compromiso. 
Se trae a consideración de los estudiantes, a través de un personaje de Vasili Grossman (2011), que uno de los efectos del encuentro con la auténtica belleza es revelar personalmente, no en abstracto, que la vida es un bien, aún en medio de situaciones tan atroces para su preservación y la convivencia como la guerra.

La belleza, se diría, invita o produce un justo olvido del propio interés, de gran riqueza moral. Puede lograr incluso que, ante ella, ni la angustia personal del dolor o el enfrentamiento prevalezcan necesariamente por encima del valor y la fraternidad que revela, y que se deben honrar con la conducta. Es pues, particularmente valiosa y necesaria para acompañar a quienes desarrollan capacidades de uso e instrumentalización de la realidad, como les ocurre de forma muy notable a los habitantes del mundo caracterizado por el consumo.

Ante la belleza, cabe la posibilidad de que se dé un reordenamiento del propio universo percibido, no en torno a uno mismo y en soledad, sino en convivencia y en relación de respeto con el valor en sí que encierra lo bello (sea en la naturaleza, en la tecnología o en la sociedad). Se logra así procurar el bien de aquello y no solo del yo, evitando una vía solitaria y voluntarista en la respuesta a la verdad mediante el compromiso.

Al hilo con lo argumentado, sostiene Milani (2015), quien es citado en esta sesión final, que "el saber mirar vincula los ámbitos estético, gnoseológico y moral para traducirse en una contemplación interior" (p. 22). Podría añadirse a sus palabras que esa vinculación la realiza el saber mirar de suyo: como por efecto del poder moral que encierra la experiencia personal profunda, no instrumental, de la realidad, a través de la puerta de acceso que ofrece su belleza. De esta manera, contemplando se aprende, en cierto modo espontáneamente, como por efecto del poder de la belleza, a vincular el ámbito más centrado en la sensibilidad ("estético"), con el más centrado en el conocimiento ("gnoseológico") y el más centrado en la voluntad, acción y compromiso ("moral").

Gadamer, por su parte, quien también es citado en esta última sesión, recuerda la magistral intuición poética de Rilke, respecto de la belleza, como impulsora del deseo de cambiar de vida, a mejor (Gadamer, 1991, pp. 8990). Y esto es lo que ha de buscar y promover la educación moral. Lejos de constituir esa propuesta un academicismo culto o inalcanzable, los alumnos tienen afortunadamente con mucha frecuencia experiencia del deseo que se ha producido en ellos de cambiar, de ser mejores, cuando se encuentran con una belleza inesperada que se lo solicita: sienten el deseo de estar a su altura. 
Desde esa experiencia, o desde su deseo, palpable en clase, y expresado también como indignación ante la injusticia o el maltrato no pocas veces, se les anima con la asignatura a que esa sea la constante moral de sus vidas: saber mirar para ver el valor en la belleza. Y, mirada con detenimiento, responder a ella personalmente, y no al dictado de ninguna autoridad que no haya pasado por el propio corazón, que es el que debe discernir siempre cada autoridad como tal o rechazarla como intrusa: la raíz de lo moral reside en el valor de la realidad.

\section{Conclusiones}

En este artículo se ha presentado un planteamiento teórico y metodológico -llevado a la práctica a través de una asignatura universitaria, pero aplicable fuera de este ámbito- cuyo objetivo es fomentar una educación moral que en última instancia favorezca comportamientos y actitudes personales, adquiridos por voluntad propia, a favor de un mundo más sostenible y justo a nivel ambiental y social. Las propuestas educativas presentadas pueden ser de interés y aplicación, sin embargo, más allá del campo de la sostenibilidad y justicia ambiental y social. Las principales ideas que se han desarrollado a lo largo del texto se presentan, de forma sintética y a modo de recapitulación, a continuación:

1. Los valores de naturaleza y sociedad son fundantes, antropológicamente anteriores a los desarrollos culturales actuales, por lo que interpelan a la conducta moral de cada persona y cultura que esté dispuesta a reconocerlos y asumirlos libremente como guía del deber ser de la propia conducta.

2. El estudio de las relaciones entre seres humanos y entre estos y la naturaleza, así como de las consecuencias que las propias decisiones libres tienen sobre los valores naturales y sociales, se estiman temas de gran interés educativo, pues interpelan a la conducta moral individual. Paralelamente, la elección de temas que presentan o abordan injusticias ambientales y sociales ante los que el alumnado, generalmente, muestra gran sensibilidad, favorece el diálogo, el debate y la reflexión personal en torno a las causas más profundas, morales, de esas injusticias.

3. Es necesario plantear modos para facilitar la obtención de respuestas personales a los interrogantes sobre el deber ser de la propia conducta, que salven el riesgo de caer en un relativismo descomprometido a la vez que garanticen la propia libertad. En este sentido, se estima más relevante ofrecer 
al alumnado herramientas -como la reflexión académica e individual en torno a temas de interés ambiental y social- con las que ir descubriendo y concluyendo los contenidos específicos de la moralidad que puedan hacer referencia a su conducta, que presentar o proponer una mera serie de conductas o contenidos culturalmente aceptados sin contemplar su aceptación personal. La educación moral debe facilitar el descubrimiento personal del valor y las llamadas a la conducta individual y a su compromiso creativo con el valor.

4. El conocimiento fragmentado o parcial de una realidad que per se es compleja, no permite alcanzar la plenitud de valor que atesora la misma, ni reconocer y enfrentar toda la gravedad de los conflictos que puede causar la conducta humana. Por tanto, es necesario abordar el estudio de la realidad, con sus dimensiones material (natural y artificial) e intangible, desde una perspectiva integradora.

5. La ausencia o debilidad del vínculo entre conocimiento y moralidad propio de numerosos desarrollos intelectuales científicos y tecnológicos actuales, se manifiesta también en el contexto del aprendizaje, pues entraña gran dificultad que las cuestiones trabajadas en clase (teoría) se traduzcan en conductas escogidas (práctica) o armonicen con ellas. Es preciso, por tanto, ayudar a hacer más evidente para los estudiantes la conexión que debe haber entre conocimiento y responsabilidad moral, exigido por el propio valor, y no por una autoridad independiente de él. El conocimiento y la reflexión explícita sobre lo que está sucediendo actualmente con los valores naturales y sociales, contribuye a fomentar una decisión más libre hacia ellos y a adoptar compromisos que, en última instancia, conduzcan al progreso hacia una sociedad y culturas más justas.

6. La belleza presenta un gran potencial para la educación moral, ya que además de encerrar un gran valor en sí misma, su ausencia puede convertirse en evidencia de un maltrato, que interpela a la actitud y conducta de cada persona. La belleza, para ser percibida en todo su alcance y fuerza moral, requiere de una educación de la mirada, un acercamiento contemplativo, en el que el propio interés pase a un segundo plano y prime el valor descubierto. En tales condiciones, la belleza invita incluso a cambiar por elección la propia vida y conducta en favor del respeto al valor que esa belleza encierra y manifiesta a la vez. Se pretende, siguiendo esa vía de la belleza, promover un despertar del corazón que busque aunar en harmonía la sensibilidad, la inteligencia y la voluntad en la libertad para el compromiso. 


\section{REFERENCIAS BIBLIOGRÁFICAS}

Alighieri, D. (2018). Comedia. Barcelona. El Acantilado.

Álvarez, P. y Vega, P. (2009). "Actitudes ambientales y conductas sostenibles. Implicaicones para la Educación Ambiental.” Revista de Psicodidáctica, 14(2), pp. 245-260.

http://www.ehu.eus/ojs/index.php/psicodidactica/article/view/727/603 (última consulta: 08.04.2019).

Artigas, M. (1979). Karl Popper: búsqueda sin término. Barcelona. Editorial Magisterio.

Astorga, A. (2009). Desayunó café, zumo y huevo, besó mis manos, pidió perdón y se murió. Diario ABC. https://www.abc.es/20091105/cultura-libros/desayunocafe-zumo-huevo-20091105.html (última consulta: 08.04.2019)

BAUman, Z. (2007). Los retos de la educación en la modernidad líquida. Barcelona. Gedisa Editorial.

Bauman, Z. (2016). Liquid Modernity. Cambridge. Polity Press.

Calvo, S. y Gutiérrez, J. (2007). El espejismo de la Educación Ambiental. Madrid. Morata.

CARson, R. (1956). El sentido del asombro (ed. 2012). Madrid. Ediciones Encuentro.

Chandra, A. (2016). El científico y el santo. Los límites de la ciencia y el testimonio de los sabios. Palma de Mallorca. José J. de Olañeta, Editor.

Chuvieco, E., Burgui, M., Da Silva, E. V., Sánchez, D., Da Silva, A., Mourao, A. y Senna, C. (2017). "Hábitos de consumo sostenible en los estudiantes universitarios de España y Brasil”, en Valores y compromisos en la conservación ambiental. Actas del I Congreso Español de Ecoética. Alcalá de Henares, Cátedra de Ética Ambiental FTPGH-UAH, pp. 112-113.

Dood, M. (2010). “The Man Who Stopped the Dessert”. https://www.imdb.com/ title/tt1694580/ (última consulta: 08.04.2019).

Dostoyevsky, F. (2004). The idiot. Londres. Penguin Books.

Eliot, T. S. (1934). The Rock. Londres. Faber\&Faber.

Escámez, J., García, R. y Pérez, C. (2003). "La educación moral ante el reto de la pobreza". Teoría de la Educación, 15, pp. 185-212. http://dx.doi.org/10.14201/ teri.3039

Espinosa, Z. (2016). "La educación moral en contextos informales". Teoría de la Educación, 28(2), pp. 53-73. https://doi.org/http://dx.doi.org/10.14201/teoredu825373

Francisco (2015). Laudato Si'. Sobre el cuidado de la Casa Común.. Madrid. EDIBESA.

GadAmer, H. G. (1991). La actualidad de lo bello. El arte como juego, símbolo y fiesta. Barcelona. Ediciones Paidós.

GFN (2017). ¿Cuál es tu Huella Ecológica? https://www.footprintcalculator.org/ (última consulta: 08.04.2019)

Grossman, V. (2011). Por una causa justa. Barcelona. Galaxia Gutenberg. 
JORDI PUIG BAGUER, ANA VILLARROYA BALLARÍN Y MARÍA CASAS JERICÓ LA EDUCACIÓN MORAL ANTE EL RETO DE LA SOSTENIBILIDAD

Ingram, C. (1991). Bad Magic: The Failure Of Technology. An Interview With Jerry Mander. The Sun. https://www.thesunmagazine.org/issues/192/bad-magicthe-failure-of-technology (última consulta: 08.04.2019)

Irazoki, F. J. (2015). Orquesta de desaparecidos. Madrid. Hiperion.

Leopold, A. (1968). A Sand County Almanac. Nueva York. Oxford University Press.

López, A. (2005). Manifiesto contra el progreso. Palma de Mallorca. José J. de Olañeta, Editor.

Louv, R. (2006). Last child in the woods. Nueva York. Algonquin Books.

Macfarlane, R. (2016). Landmarks. Inglaterra. Penguin Books.

Milani, R. (2015). El arte del paisaje. (2. ${ }^{\text {e }}$ ed.). Madrid. Biblioteca Nueva.

Montesano, C. (2016). Beyond the Label. http://www.claudiomontesanocasillas. $\mathrm{com} /$ photogallery/beyond-the-label/\#0 (última consulta: 08.04.2019)

Nietzsche, F. (1910). The will to power. An attempted transvaluation of all values. Reino Unido. T.N. Foulis.

ONU. (2017). Objetivos de Desarrollo Sostenible.

https://www.un.org/sustainabledevelopment/es/objetivos-de-desarrollo-sostenible/ (última consulta: 08.04.2019)

Ortega y Gasset, J. (1966). “El origen deportivo del Estado”, en Obras completas. Madrid, Espasa Calpe, pp. 607-624.

PNUD (2018). Índices e indicadores de desarrollo humano. Actualización estadística de 2018. Nueva York. http://hdr.undp.org/sites/default/files/2018_human_development_statistical_update_es.pdf (última consulta: 08.04.2019)

PNUMA (2016). GEO-6. Resumen de las evaluaciones regionales del sexto informe sobre las perspectivas del medio ambiente mundial: resultados principales y mensajes políticos. Nairobi. https://wedocs.unep.org/bitstream/ handle/20.500.11822/7688/-Resumen_de_las_evaluaciones_regionales_del_ sexto_informe_sobre_las_perspectivaas_del_medio_ambiente_mundial_GEO6_Resultados_principales_y_mensajes_.pdf? sequence $=3$ \&is_Allowed=y (última consulta: 08.04.2019)

Puig, J. y Casas, M. (2017). “El impacto ambiental: un despertar ético valioso para la educación”. Teoría de la Educación, 29(1), pp. 101-128. https://doi.org/http://dx.doi.org/10.14201/teoredu2017291101128

Puig, J. y ECHARRI, F. (2018). "Environmentally significant life experiences: the look of a wolf in the lives of Ernest T. Seton, Aldo Leopold and Félix Rodríguez de la Fuente”. Environmental Education Research, 24(5), pp. 678-693. https:// doi.org/https://doi.org/10.1080/13504622.2016.1259394

Puig, J., Echarri, F. y Casas, M. (2014). “Educación ambiental, inteligencia espiritual y naturaleza”. Teoría de la Educación, 26(2), pp. 115-140. https://doi. org/10.14201/teoredu2014261115140

Rilke, R. M. (1999). “Torso de Apolo Arcaico”, en: Nuevos poemas II (la otra parte de los Nuevos poemas). Madrid. Hiperion. 
Rincón, J. C. (2010). "Voluntariado y escuela: la educación cívica para la participación ciudadana a través del servicio a la comunidad”. Bordón. Revista de Pedagogía, 62(4), pp. 113-129. https://recyt.fecyt.es/index.php/BORDON/article/ view/29322/15616 (última consulta: 08.04.2019)

Salgado, S. (2004). Sahel: The End of the Road. Berkeley. University of California Press.

Saylan, C. y Blumstein, D. T. (2011). The failure of Environmental Education (and how we can fix it). California. University of California Press.

Shakespeare, W. (2015). Macbeth. Barcelona. Espasa Libros.

Thoreau, H. D. (2015). Walden. Madrid. Errata naturae.

UNESCO (2015). Informe de la UNESCO sobre la Ciencia. Resumen ejecutivo. París. https://unesdoc.unesco.org/ark:/48223/pf0000235407_spa (última consulta: 08.04.2019)

Wittgenstein, L. (2012). Tractatus logico-philosophicus. Madrid. Alianza Editorial. 